\title{
Preliminary clinical and radiographic outcomes of proximal humeral fractures: comparison of ALPS and PHILOS plating in Asian patients in Taiwan
}

Chun-Yen Chen ${ }^{1}$, Hao-Wei Chang ${ }^{1}$, Shang-Lin Hsieh ${ }^{1}$, Chien-Chun Chang ${ }^{1,2}$, Chun-Hao Tsai ${ }^{1,3}$, Yi-Wen Chen ${ }^{4,5}$, Tsung-Li Lin ${ }^{1,3,4^{*}}$ and Chin-Jung Hsu ${ }^{1,6^{*}}$ (i)

\begin{abstract}
Background: Up to $20 \%$ of proximal humeral fractures need to be treated operatively. However, numerus complications were reported by using fixed angled locking plates. The ALPS Proximal Humerus Plating System is a new design implant with novel design features.

The aim of this study was to compare the preliminary clinical outcomes and complications of proximal humeral fractures treated with either ALPS or the proximal humeral internal locking system (PHILOS) in Asian patients in Taiwan.
\end{abstract}

Methods: Between January 2016 and December 2018, 66 patients with displaced proximal humeral fractures were analyzed retrospectively, of whom 31 underwent ALPS implant treatment and 35 underwent PHILOS implant treatment. Intraoperative blood loss and operation time, postoperative Constant-Murley Shoulder Outcome (Constant-Murley) score, and complications variables were recorded for the comparison. All cases were regularly followed up for at least 1 year.

Results: The mean follow-up period was 400.8 days (range, 367-446 days). Union was achieved in 98.5\% of patients (65/66). The ALPS group yielded similar radiologic and clinical outcomes to the PHILOS plating group for treating displaced proximal humeral fractures, including operation time, intraoperative blood loss, the Constant-Murley score, and varus malunion $(P>$ 0.05 , respectively). However, the incidence of total postoperative complications in the ALPS group was significantly lower than in the PHILOS group $(P<0.05)$. There was a trend of a lower complication rate of screws/pegs protrusion, avascular necrosis, subacromial impingement, postoperative infection, and reoperation in the ALPS group, although it was not statistically significant $(P>0.05$, respectively).

Conclusion: The ALPS group yielded similar radiologic and clinical outcomes to the PHILOS plating group for displaced proximal humeral fractures, but the ALPS group had a significantly lower total rate of complications. Therefore, ALPS may be a better option for treating proximal humeral fractures. Further larger clinical studies are needed to confirm the findings presented here.

Trial registration: Retrospective study

Keywords: Proximal humerus, ALPS locking plate, PHILOS locking plate, Fracture fixation, Asian patient, Treatment outcome, Complication

\footnotetext{
*Correspondence: alilin422@yahoo.com.tw; jeffrey59835983@gmail.com

'Department of Orthopedics, China Medical University Hospital, Taichung, Taiwan

Full list of author information is available at the end of the article
}

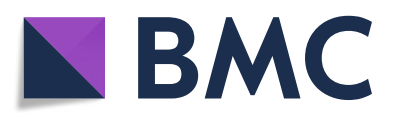

(c) The Author(s). 2020 Open Access This article is licensed under a Creative Commons Attribution 4.0 International License, which permits use, sharing, adaptation, distribution and reproduction in any medium or format, as long as you give appropriate credit to the original author(s) and the source, provide a link to the Creative Commons licence, and indicate if changes were made. The images or other third party material in this article are included in the article's Creative Commons licence, unless indicated otherwise in a credit line to the material. If material is not included in the article's Creative Commons licence and your intended use is not permitted by statutory regulation or exceeds the permitted use, you will need to obtain permission directly from the copyright holder. To view a copy of this licence, visit http://creativecommons.org/licenses/by/4.0/. The Creative Commons Public Domain Dedication waiver (http://creativecommons.org/publicdomain/zero/1.0/) applies to the data made available in this article, unless otherwise stated in a credit line to the data. 


\section{Background}

Fracture of the proximal humerus is the seventh most common cause of fracture in adults [1] and the second most common cause of upper extremity fractures, accounting for approximately 5\% of all fractures [2]. From 1990 to 2020, the age-adjusted incidence of this fracture type has increased by $15 \%$ annually, most likely because of the increasing prevalence of osteoporotic injuries and the mean age of the affected patients [3].

Although most proximal humeral fractures are treated nonoperatively, certain complex fractures require surgical treatment [4]. Surgical treatment includes use of various techniques such as intramedullary locking nail osteosynthesis, open reduction and locking plate osteosynthesis, primary hemiarthroplasty, and reverse shoulder arthroplasty [5]. Numerous surveys have suggested that angular stable implants provide sufficient fracture stabilization in older patients [6, 7]. Of these implants, the proximal humeral internal locking system (PHILOS) (Synthes, Solothurn Switzerland) is a well-established and widely reported implant with good results $[8,9]$. However, it is frequently associated with high complication rates (up to 28.2\%), such as humeral head avascular necrosis (AVN), secondary fracture displacement, screw cut-out with intraarticular penetration, or subacromial impingement with plate [10-12]. Another type of osteosynthesis, the ALPS Proximal Humerus Plating System (Zimmer Biomet, Warsaw, Indiana, USA), has novel design features, such as smooth blunt-ended pegs, multidirectional medial calcar locking screws, increased numbers of suture holes, and a lower position of the plate, to reduce the risk of the abovementioned complications [13]. However, few studies have addressed the outcome of this novel locking plate system or have compared ALPS and PHILOS for the fixation of proximal humeral fractures. In this study, we report the preliminary clinical and radiographic outcomes of ALPS treatment for proximal humeral fractures compared to PHILOS treatment in an Asian population in Taiwan.

\section{Methods}

This was a non-randomized retrospective study conducted between January 2016 and December 2018. The study was approved by the local IRB/Research Ethics Committee (approval numberCMUH102-REC2-062). A total of 66 patients with proximal humeral fractures received an open reduction and internal fixation (ORIF) using locking plates. The inclusion criteria were presence of closed proximal humeral fractures (2-, 3-, 4-part, according to the Neer classification system [14]) and age $>20$ years. Patients with glenohumeral joint dislocation, open, pathological, multiple fractures, or a follow-up period less than 1 year were excluded. Fractures were classified on the basis of preoperative plain radiographs with computed tomographic images. ORIF with ALPS or PHILOS was performed consecutively for these proximal humeral fractures. We used PHILOS in the early period and used ALPS consecutively in these fractures because ALPS was introduced after PHILOS in Taiwan. Thirtyfive patients were treated with PHILOS (PHILOS group) (Fig. $1 \mathrm{a}-\mathrm{c}$ ), and 31 patients were treated with ALPS (ALPS group) (Fig. 1d-f).

The surgery in all cases was performed by two consultants specialized in upper limb surgery (Tsung-Li Lin and Chin-Jung $\mathrm{Hsu}$ ) with patients under general anesthesia. The patients were placed in a supine position. The deltopectoral approach was applied [15]. First, the incision was made on the anterior aspect of the shoulder with exposure of the deltoid muscle. Second, the fracture was reduced, followed by the application of heavy sutures to control the rotator cuff and associated fragments. In the ALPS group, the high or low plate was chosen on the basis of the fracture pattern and to avoid impingement; proximal holes were drilled up to the subchondral bone and fixed using locking pegs rather than locking screws; then we chose the most suitable trajectory of the medial calcar screws or pegs under fluoroscopy. The remaining holes were fixed using locking screws. In both groups, the tuberosities were fixed with at least three transosseous sutures as augmentation and attached to the plate in all patients (Fig. 2).

Postoperatively, all patients underwent a similar physical therapy program. The shoulder was immobilized in an arm sling for the first 2 postoperative days. Gentle passive pendulum exercises were introduced after the suture removal to prevent shoulder stiffness. After 4 weeks, all patients began gentle passive flexion, abduction, and rotation exercises. Active exercise was prescribed after eight weeks. We reviewed patients' charts including operation notes, preoperative or postoperative radiographic reports, and out-patient department records. The operation time, operation blood loss, bone union time, postoperative functional outcomes (Constant-Murley score), and complications were analyzed at the final follow-up. All results were compared between the two groups. The continuous variables were presented as mean \pm standard deviation. The categorical variables are presented as number and percentage. The $t$ test was used to compare the differences in the continuous variables, while the Fisher's exact test was used for categorical variables between the two treatment groups. Statistical analyses were performed using SPSS for Windows version 24 (SPSS Inc., Armonk, NY). Statistical significance was set at $P<0.05$.

\section{Results}

The study cohort was comprised of 19 male and 47 female patients with a mean age of 58.9 years (range, 19- 


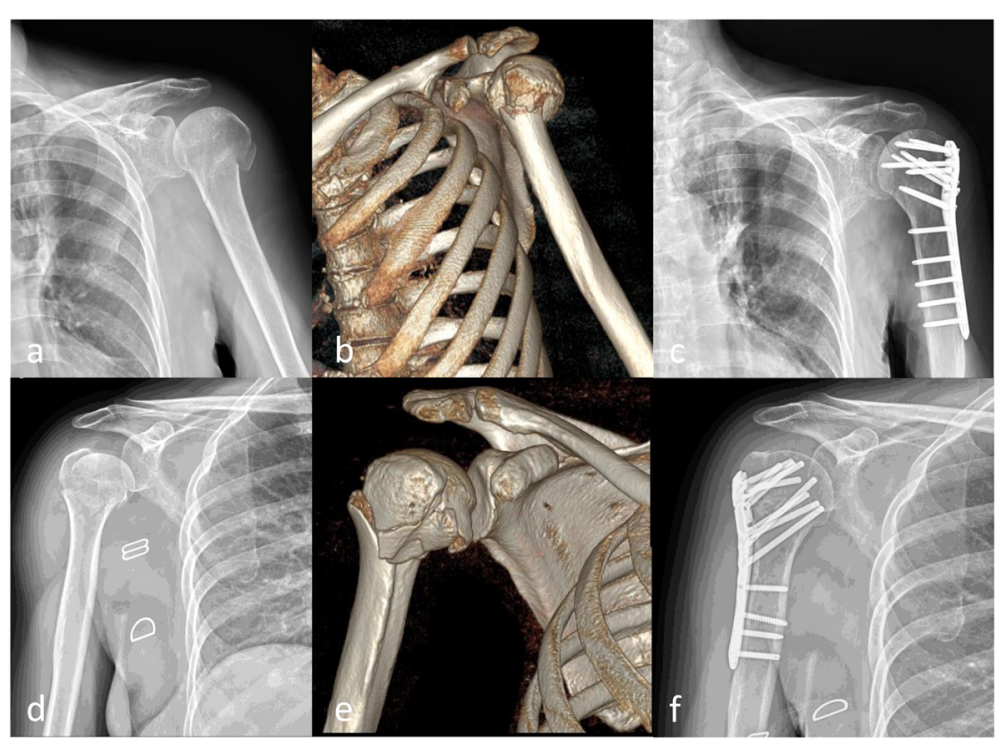

Fig. 1 a Radiograph of a 79-year-old woman with Neer classification of three-part proximal humeral fracture. b Three-dimensional (3D) computed tomography (CT) reconstruction. c Radiograph at final follow-up 360 days after surgery with PHILOS plating. $\mathbf{d}$ Radiograph of a 72-year-old woman with Neer classification of three-part proximal humeral fracture. e CT scan with 3D reconstruction. f Radiograph at final follow-up 375 days after surgery with ALPS plating

84 years). Thirty-six patients were older than 60 years and 30 patients were younger than 60 years. The traumatic mechanism for the fractures were simple falls in 28 patients and road traffic accidents in 38 patients. According to the Neer classification system, two-part

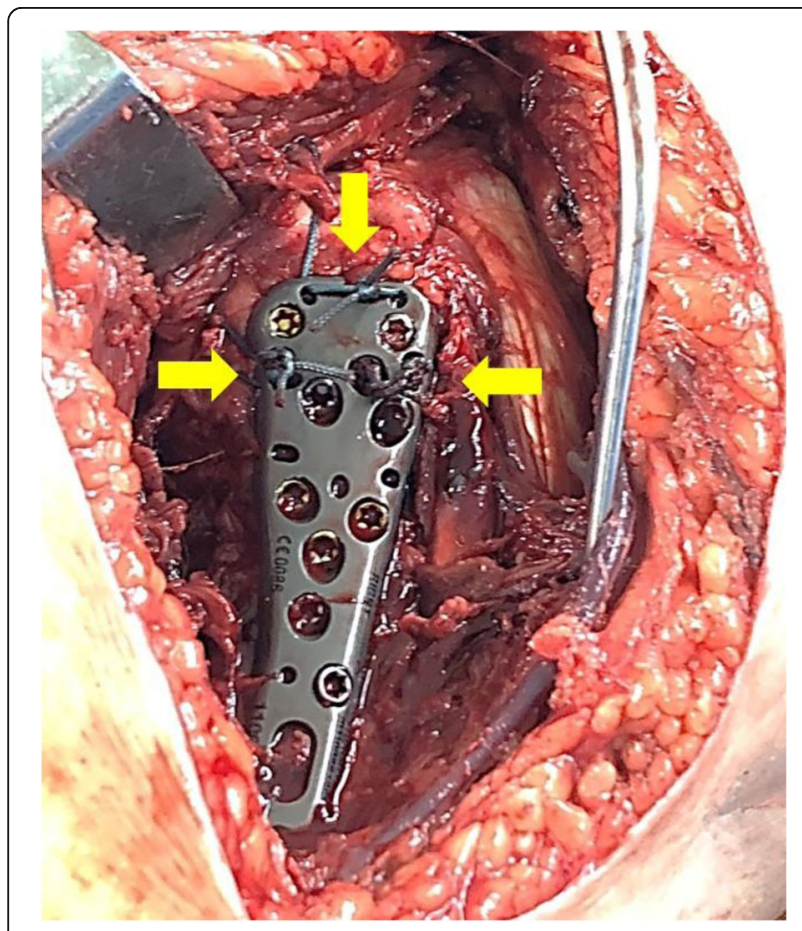

Fig. 2 Three transosseous sutures at the GT fragment (yellow arrow) attached to the high-setting ALPS plate fractures were noted in 19 patients, three-part fractures in 34 patients, and four-part fractures in 13 patients. The mean follow-up period was 400.8 days (range, 367446 days). Of the 66 patients, 65 patients (98.5\%, men, 18; women, 47) had complete bone union. Patients' demographics and radiographic findings are shown in Table 1.

In the ALPS group, the average surgery time was $137.0 \mathrm{~min}$ (range, 71-262 min), and the mean total intraoperative body blood loss was 229.6 cc (range, 10-800 cc). Two patients $(6.5 \%, 2 / 31)$ underwent surgery using low plates. The mean Constant-Murley score was 76.4 (range, 37-4). During the follow-up, varus malunion in two patients $(6.5 \%, 2 / 31)$ was noted. In the PHILOS group, the average operation time was 135.7 min (range, 83-251 mins) and the amount of blood loss was $187.1 \mathrm{cc}$ (range, 30-1150 cc). The postoperative mean ConstantMurley score was 73.2 (range, 32-95) at the last followup. Postoperative radiography on X-ray view revealed varus malunion in six patients $(17.1 \%, 6 / 35)$. There were no statistically significant differences in clinical and operative findings between the two groups $(P>0.05)$ (Table 2).

We recorded complications such as screw/peg protrusion, AVN, subacromial impingement, postoperative infection, shoulder stiffness, greater tuberosity (GT) loss reduction, or implant failure (Table 3).

In comparison with the PHILOS group, the ALPS group had a significantly lower total complication rate $(P=$ 0.049). There was a trend of lower complication rate of screws/pegs protrusion, AVN, subacromial impingement, 
Table 1 Demographic data and radiographic findings of patients

\begin{tabular}{llll}
\hline & ALPS group & PHILOS group & $P$ value \\
\hline$N$ & 31 & 35 & \\
Age in years (mean \pm SD) & $60.2 \pm 12.6$ & $56.1 \pm 17.6$ & 0.29 \\
Gender (male:female) & $06: 25$ & $13: 22$ & 0.11 \\
Mechanism (high:low energy) & $15: 16$ & $23: 12$ & 0.15 \\
Fracture type (Neer II:III:IV parts) & $07: 17: 07$ & $12: 17: 06$ & 0.56 \\
Smoking & 3 & 5 & 0.71 \\
Follow-up (days) & 405.1 & 397 & 0.81 \\
\hline
\end{tabular}

ALPS ALPS Proximal Humerus Plating System, PHILOS proximal humeral internal locking system

and postoperative infection in the ALPS group. However, differences in the rate of specific complications between the two groups were not statistically significant. Shoulder stiffness was noted in two patients in both groups. We arranged a shoulder rehabilitation program to correct the stiffness at 2 months postoperatively. The patient population did not have any cases of implant failure. Reoperation was found in three patients (two patients in PHILOS group and one patient in ALPS group). In patients treated with PHILOS, the reoperations were related to a postoperative infection $(N=1)$ and a screw protrusion $(N=1)$. In the ALPS group, one patient with GT loss reduction accepted revision surgery and bone graft augmentation with success.

\section{Discussion}

This was the first preliminary study to compare the clinical and radiographic outcomes of proximal humeral fractures treated with ALPS or PHILOS plating in Asian patients in Taiwan with at least 1 year of follow-up. Our preliminary results demonstrated that the ALPS group yielded similar radiologic and clinical outcomes to the PHILOS plating group for displaced proximal humeral fractures, including operation time, intraoperative blood loss, the Constant-Murley score, and varus malunion. However, the ALPS group resulted in a significantly lower total complication rate than the PHILOS group. There was also a trend of less complication rate of screws/pegs protrusion, AVN, subacromial impingement, postoperative infection, and reoperation in the
ALPS group, although this was not statistically significant.

Operative treatment for comminuted and displaced proximal humeral fractures is complex and challenging. The angular locking plate has demonstrated good clinical outcomes [9, 14, 16-18]; however, it is frequently associated with high complication rates (up to $28.2 \%$ ) [1012]. The main complication associated with PHILOS plating is subacromial impingement. In an early series of 28 consecutive patients, up to $21.4 \%$ of patients experienced this complication, which resulted from the superior positioning of the PHILOS plate [6]. The authors suggested that the PHILOS plate should be placed more distally. The technique of using a K-wire inserted through a hole at the top of the plate and lined up with the tip of the GT was recommended with fluoroscopy. In contrast to PHILOS, ALPS with the design of a central K-wire hole keeps the plate in a proper position more easily. Moreover, ALPS can use two types of plate options: low- and high-sitting plates. A retrospective study revealed that none of the patients in whom lowsitting plates were used had subacromial impingement [19]. The current study demonstrated the decreased rate of subacromial impingement in the ALPS group (0/31, $0 \%)$, even though most patients $(29 / 31,93.5 \%)$ were treated with high-sitting plates, compared with four cases $(4 / 35,11.4 \%)$ in the PHILOS group.

The complication of screw/peg protrusions became bothersome due to the high prevalence of osteoporotic injuries and the mean age of the affected patients. Spross et al. reported that screw/peg protrusions occurred at a rate of $11.2 \%$ with PHILOS plating [17]. Clavert et al. described a similar higher rate of screw cut-out of up to 13.7\% [12]. Moreover, a review study indicated that nearly half of the patients who experienced screw protrusions were older than 60 years, including when an angular stable locking plate was used [20]. Several studies on bone fixation using PHILOS have indicated that screw protrusion is the most common reason for revision surgery $[17,18]$. In contrast to the locking screws construct, the ALPS novel design of smooth blunt-ended pegs not only decreased the symptoms of protrusion, but also theoretically griped the bone stock effectively. However, there have been no biomechanical or clinical studies to support the design of pegs in proximal

Table 2 Radiologic, clinical, and operative results in both groups

\begin{tabular}{llll}
\hline & ALPS group & PHILOS group & $P$ value \\
\hline Operation time $(\mathrm{min})(\mathrm{mean} \pm \mathrm{SD})$ & $137.0 \pm 45.3$ & $135.7 \pm 34.5$ & 0.89 \\
Intraoperative blood loss $(\mathrm{mL})$ & $229.7 \pm 171.1$ & $187.1 \pm 192.9$ & 0.35 \\
Constant-Murley score & $76.4 \pm 13.6$ & $73.2 \pm 15.2$ & 0.37 \\
Varus malunion $n(\%)$ & $2(6.5 \%)$ & $6(17.1 \%)$ & 0.27 \\
\hline
\end{tabular}

ALPS ALPS Proximal Humerus Plating System, PHILOS proximal humeral internal locking system 
Table 3 Postoperative complications in both groups

\begin{tabular}{llll}
\hline Complications & ALPS group N (\%) & PHILOS group N (\%) & $P$ value \\
\hline Total number & $7(22.6)$ & $16(45.7)$ & 0.049 \\
Screws/pegs protrusion & $2(6.5)$ & $3(8.6)$ & $6(17.1)$ \\
AVN & $1(3.2)$ & $4(11.4)$ & 1 \\
Subacromial impingement & $0(0)$ & $1(2.9)$ & 0.11 \\
Postoperative infection & $0(0)$ & $2(5.7)$ & 0.12 \\
Shoulder stiffness & $2(6.5)$ & $0(0)$ & 1 \\
GT loss reduction & $2(6.5)$ & $0(0)$ & 1 \\
Implant failure & $0(0)$ & $2(5.7)$ & 1 \\
Reoperation & $1(3.2)$ & 0.22 \\
\hline
\end{tabular}

ALPS ALPS Proximal Humerus Plating System, AVN avascular necrosis, GT greater tuberosity, PHILOS proximal humeral internal locking system

humeral fractures. In the current study, the screw/peg protrusion rate was $6.5 \%(2 / 31)$ in the ALPS group and $8.6 \%(3 / 35)$ in the PHILOS group. All patients with this complication were older than 60 and suffered from collapse of the humeral head. There was no statistically significant difference in screw/peg protrusions between the two groups or the sub-group analysis of patients older than 60. One patient in the PHILOS group accepted revision surgery due to a screw protrusion. In the ALPS group, the two patients who suffered from peg protrusions were neither symptomatic nor had a of loss reduction. We noted that the novel design of the pegs in ALPS has a comparable effectiveness to grip the bone stock as in the PHILOS technique. Nevertheless, the use of blunt pegs prevents revision surgeries due to less irritation of the protrusion.

Although the sub-group analysis of the complication rate was not statistically significant, we observed AVN as the predominant complication in the PHILOS group.

This adverse outcome could be associated with shoulder pain, limited range of motion, and arthritis of the glenohumeral joint. Several observational studies have reported an AVN rate of 0 to $>30 \%$ in patients following PHILOS fixation $[6,10,16,18]$. Boesmueller et al. revealed that the high rate of AVN could be attributed to the use of AO (Association of Osteosynthesis) and the Neer classification systems [21]. Cadaver studies have indicated that the posterior circumflex artery is the major contributing vessel to the humeral head [22]. However, an early report related to ALPS revealed a low AVN rate [19]. The ALPS peg fixation and central K-wire hole targeting design might have reduced the AVN rate by lowering vessel injuries and improving the humeral head bone stock reserve. In our study, there were six patients (6/35, 17.1\%) who encountered AVN in the PHILOS group, which account for nearly half of the cases that developed complications. Conversely, we found that using ALPS may decrease the AVN rate (1/31, 3.2\%). However, the decrease was not significant, and further investigation of AVN is needed due to the limited sample size used in the current study.

Medial calcar screws play a key role in locking plate fixation for proximal humeral fractures [10]. The oblique locking screw placed into the inferomedial aspect of the humeral head can counteract the varus deforming force and reduce the risk of subsequent varus collapse. A prior study showed that medial calcar screws should be positioned $<12 \mathrm{~mm}$ from the apex of the arch of the calcar or within the bottom $25 \%$ of the humeral head [23]. Due to the design of the fixed angle locking plate, the fixed medial calcar screw trajectory does not match the appropriate zone in populations with different bone sizes, especially in Asian patients. Numerus studies revealed higher varus malunion rates by using angular stable locking plates such as PHILOS plating, ranging from 16 to $54.3 \%$ [20, 24, 25]. Alternatively, ALPS has an advantage of implementing multi-directional medial calcar locking screws. This may allow surgeons to have greater freedom for positioning of the calcar screw in a precise position, irrespective of plate position or bone size. For the patients with medial comminutions, the ALPS novel design of the multi-directional medial calcar may help prevent varus malunion. In the current study, there were only two patients $(6.5 \%, 2 / 31)$ who encountered varus malunion in the ALPS group compared to six patients $(17.1 \%, 6 / 35)$ in the PHILOS group. However, further clinical studies are needed to clarify this point.

Very few studies have focused on GT loss reduction of proximal humeral surgical treatments. Gillespie et al. reported a case series of 11 consecutive patients with isolated GT fractures [26], in which all patients experienced bone union without loss reduction after plate fixation. In the current study, GT loss reduction was noted in two patients in the ALPS group during the follow-up period. However, these two patients were treated with high-positioned plates, and one of the two received a further revision surgery with success. We applied PHILOS and ALPS on similarly sized sawbones to 


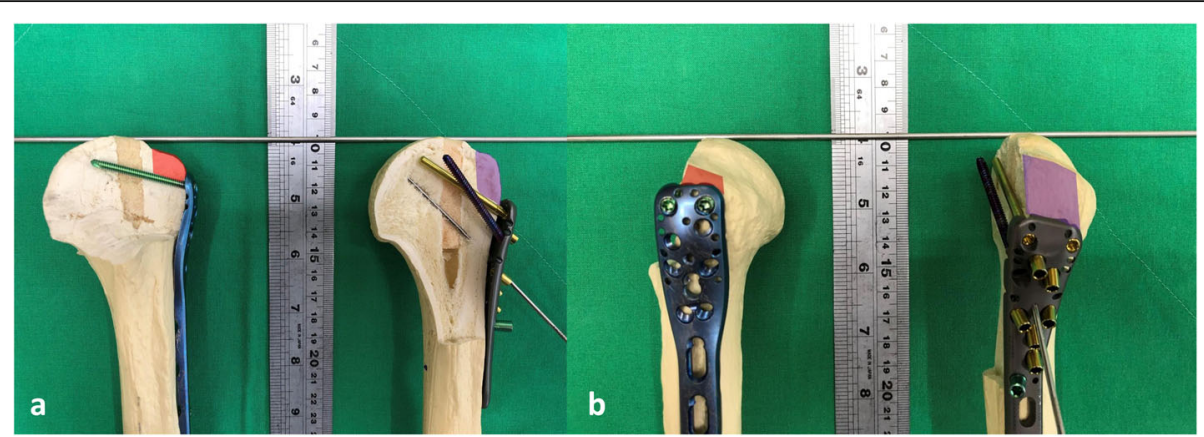

Fig. 3 For sawbones of the same size, fewer GT fragments were covered by ALPS. (Red area indicates GT fragment without PHILOS coverage; purple area, GT fragment without ALPS coverage) a Anteroposterior view of plates. b Lateral view of plates. ALPS, Proximal Humerus Plating System; GT, greater tuberosity; PHILOS, proximal humeral internal locking system

investigate the difference. The positions of PHILOS and ALPS were assessed according to the manufacturer. The results revealed that the distance between the GT and the apex of the plate was larger with ALPS than with PHILOS (Fig. 3). Although ALPS is designed to lower the complication rates of subacromial impingement, less buttressing coverage of the GT fragment was presented on the sawbone. Therefore, multiple transosseous sutures on the GT fragment should be considered for patients who are susceptible to fixation failure, such as those with low local bone mass density, increased age, and multifragmentary fracture patterns [27].

We acknowledge some limitations of this study. First, our study included a small sample size and was a retrospective study without randomization. Second, the follow-up period was relatively short in both groups; however, the average follow-up period was 1-2 years and did not justify forecasting of the long-term outcomes. Larger clinical studies are required to validate the findings presented here.

\section{Conclusions}

The ALPS group yielded similar radiologic and clinical outcomes to the PHILOS plating group for displaced proximal humeral fractures, but the ALPS group had a significantly lower total rate of complications. Therefore, ALPS may be a more favorable treatment option than PHILOS for proximal humeral fractures. Further control based multi-center randomized control trials are required in the future to confirm the efficacy and potential advantages presented here.

\section{Abbreviations \\ ALPS: ALPS Proximal Humerus Plating System; PHILOS: Proximal humeral internal locking system; AVN: Avascular necrosis; GT: Greater tuberosity; ORIF: Open reduction and internal fixation; AO: Association of Osteosynthesis; CT: Computed tomography}

\section{Acknowledgements}

This work was supported by the Department of Orthopedic Surgery, China Medical University hospital in Taichung, Taiwan.
Authors' contributions

CYC contributed to the collection of data and the writing the manuscript. $T L L$ and $C J H$ contributed to the study design and performed the surgery. HWC and YWC contributed to the data analysis. SLH, CCC, and CHT contributed to the modification of the manuscript. All authors have read and approved the final manuscript.

\section{Funding}

This research received no specific grant from any funding agency in the public, commercial, or not-for-profit sectors.

Availability of data and materials

The dataset supporting the conclusions of this article is included within the article.

Ethics approval and consent to participate

The study was approved by the local IRB/Research Ethics Committee, CMUH102-REC2-062.

\section{Consent for publication}

Written informed consent was obtained from the patients for the publication of this report and any accompanying images.

\section{Competing interests}

The authors declare that they have no competing interests.

\section{Author details}

${ }^{1}$ Department of Orthopedics, China Medical University Hospital, Taichung, Taiwan. ${ }^{2}$ Ph.D. Degree Program of Biomedical Science and Engineering, National Chiao Tung University, Hsinchu, Taiwan. ${ }^{3}$ Department of Sports Medicine, China Medical University, Taichung, Taiwan. ${ }^{4}$ Graduate Institute of Biomedical Sciences, China Medical University, Taichung, Taiwan. ${ }^{5}$ 3D Printing Medical Research Institute, Asia University, Taichung, Taiwan. ${ }^{6}$ School of Chinese Medicine, China Medical University, Taichung, Taiwan.

Received: 14 June 2020 Accepted: 30 July 2020

Published online: 28 August 2020

\section{References}

1. Roux A, Decroocq L, El Batti S, Bonnevialle N, Moineau G, Trojani C, Boileau $P$, de Peretti F. Epidemiology of proximal humerus fractures managed in a trauma center. Orthop Traumatol Surg Res. 2012;98(6):715-9.

2. Lind $T$, Krøner $K$, Jensen J. The epidemiology of fractures of the proximal humerus. Arch Orthop Trauma Surg. 1989;108(5):285-7.

3. Kannus P, Palvanen M, Niemi S, Parkkari J, Järvinen M, Vuori I. Osteoporotic fractures of the proximal humerus in elderly Finnish persons: sharp increase in 1970-1998 and alarming projections for the new millennium. Acta Orthop Scand. 2000;71(5):465-70.

4. Schumaier A, Grawe B. Proximal humerus fractures: evaluation and management in the elderly patient. Geriatr Orthop Surg Rehabil. 2018;9: $1-11$. 
5. Maier D, Jaeger M, Izadpanah K, Strohm PC, Suedkamp NP. Proximal humeral fracture treatment in adults. J Bone Joint Surg Am. 2014;96(3):251-61.

6. Geiger EV, Maier M, Kelm A, Wutzler S, Seebach C, Marz I. Functional outcome and complications following PHILOS plate fixation in proximal humeral fractures. Acta Orthop Traumatol Turc. 2010;44(1):1-6.

7. Fankhauser F, Boldin C, Schippinger G, Haunschmid C, Szyszkowitz R. A new locking plate for unstable fractures of the proximal humerus. Clin Orthop Relat Res. 2005;430:176-81.

8. Moonot P, Ashwood N, Hamlet M. Early results for treatment of three- and four-part fractures of the proximal humerus using the PHILOS plate system. J Bone Joint Surg Br. 2007;89(9):1206-9.

9. Gavaskar AS, Karthik BB, Tummala NC, Srinivasan P, Gopalan H. Second generation locked plating for complex proximal humerus fractures in very elderly patients. Injury. 2016;47(11):2534-8.

10. Schliemann B, Wähnert D, Theisen C, Herbort M, Kösters C, Raschke MJ, Weimann A. How to enhance the stability of locking plate fixation of proximal humerus fractures? An overview of current biomechanical and clinical data. Injury. 2015;46(7):1207-14..

11. Gupta AK, Harris JD, Erickson BJ, Abrams GD, Bruce B, McCormick F, Nicholson GP, Romeo AA. Surgical management of complex proximal humerus fractures-a systematic review of 92 studies including 4500 patients. J Orthop Trauma. 2015;29(1):54-9.

12. Clavert P, Adam P, Bevort A, Bonnomet F, Kempf JF. Pitfalls and complications with locking plate for proximal humerus fracture. J Shoulder Elbow Surg. 2010;19(4):489-94.

13. Bai $L, F u Z$, An S, Zhang $P$, Zhang D, Jiang B. Effect of calcar screw use in surgical neck fractures of the proximal humerus with unstable medial support: a biomechanical study. J Orthop Trauma. 2014;28(8):452-7.

14. Neer CS. Displaced proximal humeral fractures. I. Classification and evaluation. J Bone Joint Surg Am. 1970;52(6):1077-89.

15. Zlotolow DA, Catalano LW, Barron OA, Glickel SZ. Surgical exposures of the humerus. J Am Acad Orthop Surg. 2006;14(13):754-65.

16. Faraj D, Kooistra BW, Vd Stappen WAH, Werre AJ. Results of 131 consecutive operated patients with a displaced proximal humerus fracture: an analysis with more than two years follow-up. Eur J Orthop Surg Traumatol. 2011; 21(1):7-12.

17. Spross C, Platz A, Rufibach K, Lattmann T, Forberger J, Dietrich M. The PHILOS plate for proximal humeral fractures-risk factors for complications at one year. J Trauma Acute Care Surg. 2012;72(3):783-92.

18. Sproul RC, lyengar JJ, Devcic Z, Feeley BT. A systematic review of locking plate fixation of proximal humerus fractures. Injury. 2011;42(4):408-13.

19. Argyropoulos M, Ken M. Early results of the A.L.P.S. proximal humerus locking plate. Open Orthop J. 2018;12:53-8.

20. Owsley KC, Gorczyca JT. Fracture displacement and screw cutout after open reduction and locked plate fixation of proximal humeral fractures [corrected]. J Bone Joint Surg Am. 2008;90(2):233-40.

21. Boesmueller S, Wech M, Gregori M, Domaszewski F, Bukaty A, Fialka C, Albrecht C. Risk factors for humeral head necrosis and non-union after plating in proximal humeral fractures. Injury. 2016;47(2):350-5.

22. Hettrich CM, Boraiah S, Dyke JP, Neviaser A, Helfet DL, Lorich DG. Quantitative assessment of the vascularity of the proximal part of the humerus. J Bone Joint Surg Am. 2010;92(4):943-8.

23. Padegimas EM, Zmistowski B, Lawrence C, Palmquist A, Nicholson TA, Namdari S. Defining optimal calcar screw positioning in proximal humerus fracture fixation. J Shoulder Elbow Surg. 2017;26(11):1931-7.

24. Helwig P, Bahrs C, Epple B, Oehm J, Eingartner C, Weise K. Does fixed-angle plate osteosynthesis solve the problems of a fractured proximal humerus? A prospective series of 87 patients. Acta Orthop. 2009;80(1):92-6.

25. Solberg BD, Moon CN, Franco DP, Paiement GD. Locked plating of 3- and 4part proximal humerus fractures in older patients: the effect of initial fracture pattern on outcome. J Orthop Trauma. 2009;23(2):113-9.

26. Gillespie RJ, Johnston PS, Gordon VA, Ward PJ, Getz CL. Using plate osteosynthesis to treat isolated greater tuberosity fractures. Am J Orthop (Belle Mead NJ). 2015;44(8):E248-51.

27. Krappinger D, Bizzotto N, Riedmann S, Kammerlander C, Hengg C, Kralinger FS. Predicting failure after surgical fixation of proximal humerus fractures. Injury. 2011:42(11):1283-8.

\section{Publisher's Note}

Springer Nature remains neutral with regard to jurisdictional claims in published maps and institutional affiliations.

Ready to submit your research? Choose BMC and benefit from:

- fast, convenient online submission

- thorough peer review by experienced researchers in your field

- rapid publication on acceptance

- support for research data, including large and complex data types

- gold Open Access which fosters wider collaboration and increased citations

- maximum visibility for your research: over $100 \mathrm{M}$ website views per year

At BMC, research is always in progress.

Learn more biomedcentral.com/submissions 